\title{
Моделирование процесса теплопроводности с использованием систем клеточных автоматов
}

\author{
С.П. Бобков 1, д.m.н., npoфpeccop, bsp@isuct.ru \\ Э.Г. Галиаскаров ${ }^{1}$, к.х.н., доиент \\ 1 Ивановский государственный химико-технологический университет, \\ г. Иваново, 15300о, Россия
}

Статья посвящена вопросам использования дискретных динамических моделей в качестве альтернативы классическим методам исследования тепловых процессов химической технологии.

Адекватное описание явлений при передаче тепла является чрезвычайно важной задачей как в теоретическом плане, так и с позиций практического использования тепловых процессов. Кроме того, современные методы обучения требуют внедрения электронных учебников, виртуальных лабораторных практикумов, моделирующих программ, которые также нуждаются в корректном описании исследуемых явлений.

Классический подход к моделированию передачи тепла в сплошной среде предполагает использование уравнений теплопроводности, в которых теплофизические характеристики материалов обычно являются константами. Учет влияния температуры на характеристики материалов приводит к необходимости исследовать нелинейные уравнения, что вызывает значительные вычислительные сложности. В связи с этим целесообразно использовать принципиально иные подходы к моделированию теплопроводности, одним из которых являются модели на основе систем клеточных автоматов.

В работе использованы дискретные динамические модели в виде систем детерминированных клеточных автоматов. При этом сплошная среда рассматривается как совокупность взаимодействующих элементов, поведение которых полностью описывается локальными функциями. Описаны основные подходы и общая методология разработки дискретных моделей.

Рассмотрены примеры использования систем клеточных автоматов для моделирования нелинейных процессов теплообмена с учетом неоднородности материала и наличия в нем объемных источников переменной мощности.

Полученные данные дискретного моделирования хорошо согласуются с результатами использования классического подхода и не противоречат общепринятым взглядам, принятым в теории тепловых явлений. В работе показаны особенности дискретного подхода в сравнении с применением уравнений в частных производных с нелинейными коэффициентами, указаны достоинства и недостатки.

Ключевые слова: дискретное моделирование, клеточные автоматы, теплопередача, нелинейные задачи теплопроводности, объемные источники тепла.

При разработке и совершенствовании оборудования химической технологии, как и во всех современных областях науки, широко применяются методы математического моделирования. Следует отметить, что базовыми процессами здесь считаются гидродинамические, тепловые, диффузионные, механические, то есть с общепринятых позиций это процессы в сплошной среде. В этом случае подходы к их моделированию предполагают использование уравнений математической физики, которые являются формализацией фундаментальных законов переноса субстанции (энергии, массы). Они обычно записываются в виде дифференциальных уравнений с частными производными параболического или гиперболического типа [1]. Данные уравнения считаются классическими. Их применение внесло огромный вклад в становление и развитие химической инженерной науки. Тем не менее, современные исследователи стали обращать внимание на недостатки классических подходов, которые связаны, в первую очередь, с их математическими схемами, предполагающими использование бесконечно малых (производных, дифференциалов). Безусловно, использование методов математической физики ни в коей мере не является основанием для критики. Однако приверженцы классических подходов не акцентируют внимание на том, что, используя компьютеры и численные методы, они переходят от непрерывного представления задачи к дискретному со всеми вытекающими последствиями.

Далее при выводе большинства классических уравнений использовались градиентные законы переноса, связывающие соответствую- 
щие потоки и потенциалы. Последнее привело к тому, что большинство транспортных коэффициентов (теплопроводности, диффузии, вязкости и пр.) представлены в данных уравнениях константами, хотя хорошо известно, что они таковыми не являются. Практика показала, что применение классических подходов не только может приводить к недостаточно адекватным результатам, но в ряде случаев затруднено [2-6]. Это касается следующих ситуаций.

1. Исследование процессов в гетерогенных и анизотропных средах, когда свойства веществ меняются в пространстве или во времени. При этом приходится прибегать к решению последовательностей кусочно-линейных задач, вводя упрощающие допущения.

2. Наличие в дифференциальных уравнениях нелинейных или пороговых функций. В таких обстоятельствах получить аналитические решения практически невозможно, а численные методы либо плохо распараллеливаются (неявные схемы решений), либо ограничены условиями устойчивости и точности (явные схемы).

3. Сложная геометрия объекта моделирования - несимметричные области, криволинейные границы. Здесь проблемы возникают как при описании самой исследуемой области, так и при постановке граничных условий в двух- и трехмерных случаях.

Указанные проблемы существенно ограничивают применение классических континуальных подходов при современных исследованиях основных процессов химической технологии. Одним из направлений исследований указанных процессов может быть имитационное моделирование с использованием дискретных динамических систем [7-11].

Многие исследователи при моделировании целого ряда процессов и систем обратились к новым подходам, в основу которых положен язык дискретных сред. Это агентные системы, клеточные структуры, системы взаимодействующих тьюрмитов и пр. [12, 13]. В настоящее время еще нет качественной теории этих систем, но имеется много примеров их успешного применения при решении конкретных задач. Перспективность использования дискретных методов основана на предположении: глобальная структура и поведение системы определяются локальными взаимодействиями составляющих ее элементов [2]. Дискретные методы в ряде случаев оказываются более универсальными, чем описание объекта системами дифференциальных уравнений.
Остановимся на одной из разновидностей дискретных динамических моделей - системах клеточных автоматов.

\section{Формализация клеточного автомата}

Система клеточных автоматов - дискретная динамическая модель, представляющая собой совокупность взаимодействующих пространственных элементов (клеток, ячеек). Клетки образуют пространственную решетку, размерность и формы которой в общем случае произвольные [9, 12]. Каждая клетка функционирует по правилам, напоминающим законы работы абстрактного автомата, то есть каждое новое состояние клетки в произвольный момент времени определяется ее предыдущим состоянием и, как правило, состояниями клеток-соседей. Поэтому можно считать, что каждая дискретная клетка (автомат) связана своими входами с выходами соседних клеток и выходные сигналы каждой клетки являются входными для окрестных клеток

Системы клеточных автоматов характеризуются следующими общими свойствами [12]:

- пространство состояний системы дискретно и конечно;

- изменение состояний всех клеток системы происходит одновременно;

- на конкретную клетку способны повлиять лишь соседние клетки.

Последнее свойство является очень важным при моделировании процессов переноса. Оно показывает, что поведение системы клеточных автоматов полностью определяется взаимодействиями его элементов. Это относится и к большому классу непрерывных динамических систем, описываемых уравнениями в частных производных.

Рассматривая функционирование отдельной клетки в терминах теории конечных автоматов [14, 15], необходимо указать следующее.

Каждая клетка представляет собой объект, функционирующий по шагам дискретного времени $t_{0}<t_{1}<t_{2}<\ldots$, принадлежащим множеству Г. В конкретный момент времени $t_{k} \in \Gamma$ клетка находится в одном из возможных состояний $z\left(t_{k}\right)$, принадлежащих множеству $Z$. На клетку могут воздействовать входные сигналы $X\left(t_{k}\right)$, на которые она реагирует следующим образом.

Во-первых, состояние клетки изменяется в соответствии с одношаговой функцией переходов:

$$
z\left(t_{k+1}\right)=F\left[z\left(t_{k}\right), X z\left(t_{k}\right)\right] .
$$


Во-вторых, клетка формирует выходные сигналы $Y\left(t_{k+1}\right)$, определяемые функцией выходов:

$$
Y\left(t_{k+1}\right)=\varphi\left[z\left(t_{k}\right), X z\left(t_{k}\right)\right]
$$

По характеру отсчета модельного времени каждую клетку системы следует считать синхронным автоматом, поскольку моменты $t_{0}, t_{1}$, $\ldots, t_{\mathrm{k}}$ определяются принудительно. Реакция клетки в соответствии с функциями (1) и (2) заканчивается за один шаг синхронизации.

\section{Общая методология моделирования}

Рассмотрим общую методологию моделирования процессов переноса с использованием клеточных автоматов [16].

Прежде всего непрерывное модельное пространство разбивается на клетки по функциональному признаку. Для разбиения можно использовать равномерную решетку, но это необязательно и часто делается лишь для удобства моделирования. Основной целью дискретизации пространства является получение функционально однородных клеток, то есть их размеры должны быть такими, чтобы параметры протекающих внутри процессов можно было считать не зависящими от пространственных координат. При этом единственной независимой переменной для описания поведения каждой клетки останется время. Следует заметить, что описанный подход к разбиению модельного пространства позволяет заполнить небольшими клетками области произвольной формы. Поэтому значительно уменьшаются трудности, возникающие при моделировании объектов с криволинейными границами.

Здесь же надо решить вопрос о количестве клеток-соседей, поскольку это потребуется при описании взаимного влияния элементов модели [12]. При использовании клеточных автоматов традиционно рассматриваются следующие понятия: окрестность фон Неймана - совокупность клеток, имеющих с рассматриваемой клеткой общую сторону, окрестность Мура клетки, имеющие общую вершину.

После дискретизации модельного пространства следует описать поведение полученных клеток с помощью функциональных зависимостей вида (1)-(2). Для этого следует указать явный вид функций $F$ и $\varphi$. Зависимости должны формально связывать состояния клеток и воздействия на них. При этом применяются общие законы конкретного моделируемого процесса.

При моделировании процессов переноса в качестве состояний клеток целесообразно ис- пользовать интенсивные величины - потенциальные фазовые переменные соответствующего процесса. Так, для тепловых процессов состояние клетки может однозначно определяться температурой. При моделировании диффузионных процессов состояние клетки задается концентрацией целевого компонента. В гидродинамике роль состояния играет средняя скорость движения и т.д. В такой постановке входными внешними воздействиями (входными сигналами) будут являться соответствующие экстенсивные величины - поток тепла, поток массы, механическая сила и т.д. Таким образом, будет получен взаимосвязанный массив клеток, поведение которых во времени (в данном случае дискретном) будет подчиняться законам моделируемого процесса.

Нужно отметить, что используемые функции (1) и (2) не всегда являются детерминированными и в ряде случаев могут иметь стохастический характер.

После формирования массива клеток и определения законов их функционирования вида (1) и (2) следует задать величины необходимых констант, характеризующих параметры конкретного процесса и исходные состояния клеток. Процесс моделирования будет состоять в определении значений элементов массива для последовательных моментов дискретного времени.

Важным вопросом, который необходимо решать при создании клеточно-автоматных моделей, является правильный выбор шагов квантования модельных пространства и времени. Дискретизация модельного пространства, как было указано выше, основана на получении функционально однородных клеток. Квантование времени должно происходить с учетом принятой в данном виде модели гипотезы о локальном взаимодействии клеток. Это означает, что суммарный поток субстанции (массы или энергии) от клетки к соседям не должен превышать удельное содержание данной субстанции в клетке-источнике. Эти соображения позволяют вычислить максимальную величину кванта модельного времени.

\section{Построение дискретной модели}

Среди базовых процессов химической технологии распространены процессы, у которых потенциал переноса является скалярной величиной. Это перенос тепловой энергии теплопроводностью или перенос массы диффузией. В обоих случаях имеет место молекулярный 
механизм передачи субстанции, а классические модели используют параболические дифференциальные уравнения в частных производных.

В качестве примера рассмотрим двухмерную клеточно-автоматную модель молекулярного теплопереноса [17]. Возьмем пластину произвольной формы и разобьем ее на дискретные элементы (клетки) с шагом $h$ (рис. 1$)$.

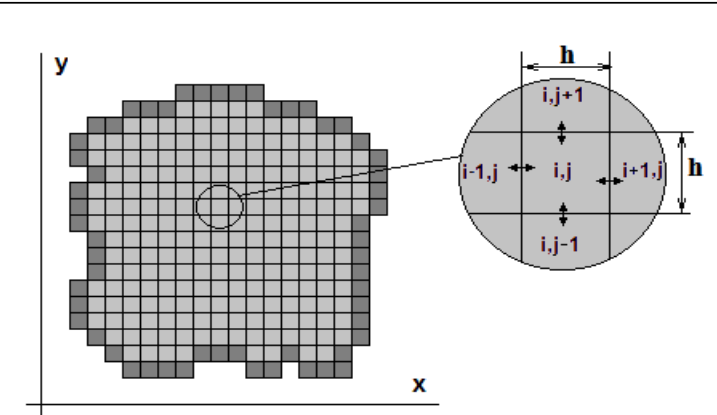

Рис. 1. Схема дискретизации объекта моделирования

Fig. 1. Modeling object sampling scheme

Каждую клетку с координатами (номером) $i, j$ будем рассматривать как конечный автомат, состояния которого характеризуются значениями температуры $T_{i j}$, а входными сигналами будут являться потоки тепла $q$ между клеткой и ее соседями. В качестве соседей будут выступать клетки, соответствующие окрестности фор Неймана.

Для определения явного вида функций (1) и (2) воспользуемся известными положениями физики.

В момент времени $t_{k}$ произвольная клетка $i, j$ обменивается с соседними клетками потоками тепла (показаны на выделенном фрагменте рис. 1). Используя интегральную форму закона теплопроводности, можно записать выражение для потока тепла, получаемого клеткой $i, j$ от клетки $i-1, j$, следующим образом:

$$
q_{1}\left(t_{k}\right)=\lambda_{i-1, j} \frac{\left[T_{i-1, j}\left(t_{k}\right)-T_{i, j}\left(t_{k}\right)\right]}{h^{2}},
$$

где $q$ - удельная мощность теплового потока; $\lambda_{i-1, j}$ - коэффициент теплопроводности материала клетки $i-1, j ; T_{i-1, j}\left(t_{k}\right)$ и $T_{i, j}\left(t_{k}\right)$ - температуры (состояния) клеток в момент времени $t_{k} ; h$-шаг по координатам.

Для потока тепла от клетки $i, j$ к клетке $i+1, j$ справедливо уравнение

$$
q_{2}\left(t_{k}\right)=\lambda_{i, j} \frac{\left[T_{i, j}\left(t_{k}\right)-T_{i+1, j}\left(t_{k}\right)\right]}{h^{2}},
$$

где $\lambda_{i, j}-$ коэффициент теплопроводности материала клетки $i, j ; T_{i+1, j}\left(t_{k}\right)$ - температура клетки $i+1, j$.

Аналогично для потоков по оси $\mathrm{Y}$ :

$$
\begin{aligned}
& q_{3}\left(t_{k}\right)=\lambda_{i, j-1} \frac{\left[T_{i, j-1}\left(t_{k}\right)-T_{i, j}\left(t_{k}\right)\right]}{h^{2}}, \\
& q_{4}\left(t_{k}\right)=\lambda_{i, j} \frac{\left[T_{i, j}\left(t_{k}\right)-T_{i, j+1}\left(t_{k}\right)\right]}{h^{2}} .
\end{aligned}
$$

Таким образом, с учетом (3)-(6) суммарный поток молекулярного переноса тепла для рассматриваемой клетки $i, j$ в момент дискретного времени $t_{k}$ будет иметь вид

$$
\sum q_{i, j}\left(t_{k}\right)=q_{1}\left(t_{k}\right)-q_{2}\left(t_{k}\right)+q_{3}\left(t_{k}\right)-q_{4}\left(t_{k}\right) .
$$

Уравнение (7) описывает входные воздействия (сигналы) на отдельную клетку-автомат. Зависимость для функции переходов можно получить, рассматривая изменение температуры клетки за промежуток времени $\Delta t$. Можно записать следующее выражение:

$$
\frac{T_{i, j}\left(t_{k+1}\right)-T_{i, j}\left(t_{k}\right)}{\Delta t}=\frac{\sum q_{i, j}\left(t_{k}\right)}{C_{i, j} \rho_{i, j}},
$$

где $C_{i, j}$ и $\rho_{i, j}$ - теплоемкость и плотность материала клетки $i, j$ соответственно.

Окончательно получим

$$
T_{i, j}\left(t_{k+1}\right)=T_{i, j}\left(t_{k}\right)+\frac{\Delta t}{C_{i, j} \rho_{i, j}} \sum q_{i, j}\left(t_{k}\right) .
$$

Выражение (8) является функцией переходов клетки, то есть позволяет вычислить новое состояние клетки - ее температуру на каждом шаге дискретного времени. Состояния клеткиавтомата можно считать также ее выходными сигналами, что, согласно теории вычислений, характерно для автоматов Мура.

Нетрудно заметить, что полученные уравнения позволяют моделировать процесс в объекте, теплофизические свойства которого неоднородны. На самом деле характеристики вещества входят в локальные зависимости (7)-(8), что уже дает возможность учитывать пространственную неоднородность. Если же свойства материала меняются во времени или зависят от температуры (состояния клетки), то нетрудно учесть эти изменения на каждом шаге по времени, введя дополнительные выражения. С этих позиций моделирование процесса в однородном материале будет являться частным случаем.

Строго говоря, выражения (7) и (8) справедливы для внутренних клеток автомата, то есть для клеток, имеющих четырех соседей. Однако при моделировании реальных процессов в объектах конечных размеров на рассмотрении бу- 
дут находиться также клетки, имеющие меньшее число соседей, то есть находящиеся на краях рассматриваемой области (на рисунке 1 выделены цветом). Получить функции перехода вида (8) для них нетрудно, если использовать методику, приведенную выше. Рассматривая потоки тепла для краевых клеток, можно выделить два случая. Если можно принять, что теплообмен с окружающей средой отсутствует, то соответствующее слагаемое выражения (7) необходимо приравнять нулю. При наличии теплообмена с внешним пространством соответствующий поток следует учитывать, а его величину определять исходя из уравнений теплоотдачи на границе сред.

Наконец, исследуемое пространство может содержать особые клетки, моделирующие участки объекта, где теплота возникает или поглощается, то есть локальные источники (стоки) тепла. Их использование в модели наиболее удобно, когда для них известен закон изменения состояния (температуры) во времени:

$$
T_{m, n}\left(t_{k}\right)=\Psi\left(t_{k}\right)
$$

где $m, n$ - номер клетки; $T_{m, n}\left(t_{k}\right)$ - температура клетки; $\Psi\left(t_{k}\right)$ - заданная функция дискретного времени, в общем случае также функция свойств материала.

Правая часть выражения (9) в ряде случаев может быть равна константе. Размеры локального источника (стока) тепла могут не ограничиваться одной клеткой, а иметь конкретные размеры и форму. Иногда в объекте моделирования содержатся не локальные, а распределенные источники (стоки) тепла, например, при объемных химических реакциях. Тогда целесообразно в правую часть уравнения (8) ввести дополнительное слагаемое, учитывающее выделение (поглощение) тепловой энергии:

$$
T_{i, j}\left(t_{k+1}\right)=T_{i, j}\left(t_{k}\right)+\frac{\Delta t}{C_{i, j} \rho_{i, j}}\left[\sum q_{i, j}\left(t_{k}\right)+\gamma\left(t_{k}\right)\right],
$$

где $\gamma\left(t_{k}\right)$ - удельная мощность источника (стока) тепла в момент времени $t_{k}$.

Безусловно, величина удельной мощности может зависеть от параметров процесса, свойств материала и пр. Некоторые конкретные виды данной зависимости будут рассмотрены далее в примерах.

\section{Примеры использования дискретного подхода}

Рассмотрим результаты моделирования процесса теплопроводности.

\section{Пример 1.}

Сначала рассмотрим тривиальную задачу прогрева однородной пластины точечным источником тепла постоянной температуры. Решение такой задачи традиционными методами не представляет трудностей, но данный пример может быть полезен для дальнейших рассуждений.

В качестве модельного объекта возьмем плоскую пластину правильной формы, которая разбита на $1681(41 \times 41)$ клетку с шагом 1 мм. Теплофизические характеристики материала следующие: удельная теплоемкость - 1000

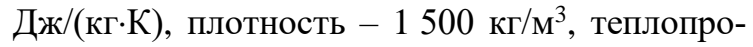
водность $-1,5$ Вт/(м·К). В исходном состоянии начальная температура среды и пластины принята равной 0 условных градусов. Источник тепла локализован в центре пластины. Температура источника постоянна и равна 5 условным градусам. Величина шага моделирования по времени равна 0,005 с. При имитации процесса предполагалось, что теплоотдача в окружающую среду отсутствует.

Результаты моделирования с использованием зависимостей (3)-(8), представленные как значения температуры в различные моменты времени, приведены на рисунке 2 (время на этом рисунке и последующих проставлено в правом верхнем углу, по горизонтальным осям отложены размеры пластины, по оси аппликат - температура в условных единицах).

Представленные результаты примера 1 свидетельствуют о постепенном прогреве пластины с асимптотическим приближением тем-

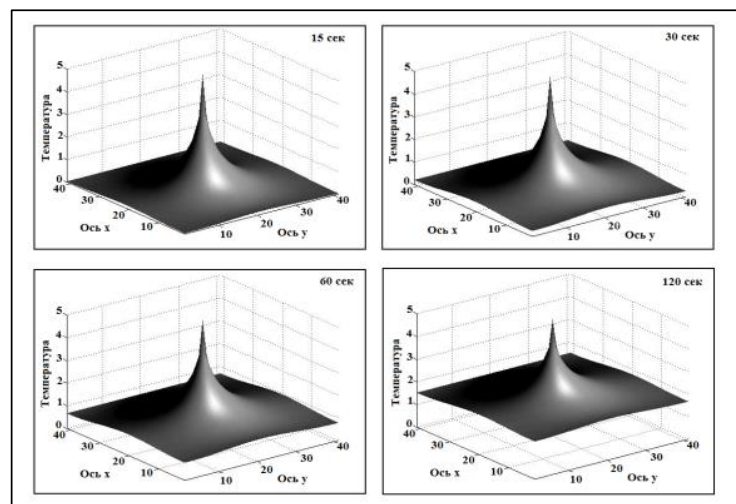

Рис. 2. Результаты исследования линейной модели проиесса теплопроводности с источником постоянной температуры в центтре пластины

Fig. 2. Results of studying a linear model of the heat conduction process with a constant temperature source in the center of the plate 
пературы к постоянному значению, равному температуре источника. Такое протекание процесса полностью соответствует существующим представлениям о природе теплопереноса.

\section{Пример 2.}

Рассмотрим имитацию процесса горения. Для этого необходимо ввести в рассмотрение объемный источник тепла и вместо выражения (8) использовать зависимость (10).

Примем, что для любого момента времени и для любой клетки выполняется следующая зависимость:

$\gamma(T)=k T$,

где $k$ - константа.

Таким образом, приходим к квазилинейной задаче, в которой удельная мощность источников зависит от температуры. Такие условия характерны для теплопереноса, осложненного экзотермическими явлениями.

При моделировании предполагалось, что для инициирования горения центральная точка пластины «поджигается» мгновенным тепловым импульсом, температура которого превышает исходную температуру пластины. Все характеристики материала и параметры моделирования были аналогичными первому примеру. Константа в выражении (11) принята равной 0,025. Результаты моделирования представлены на рисунке 3.

Очевидно, что на начальном этапе процесса тепло распространяется от более нагретых участков достаточно медленно. Но затем в области воздействия начального импульса температура резко возрастает, образуя своего рода факел. Такая картина в реальных условиях ха-

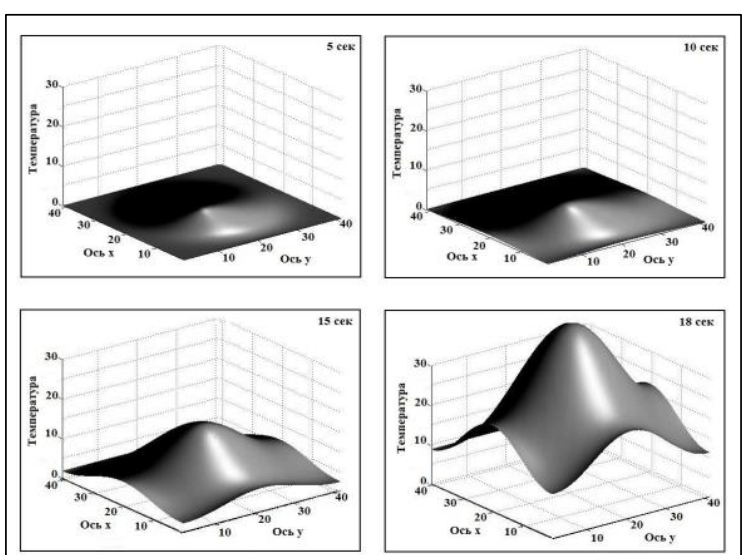

Рис. 3. Результаты исследования квазилинейной модели теплопроводности, учитывающей экзотермические эффекты

Fig. 3. The study results of a quasilinear thermal conductivity model that takes into account exothermic effects рактерна для начальных периодов процесса горения.

Пример 3.

Представляет интерес теплоперенос в неоднородной пластине, которая имеет участки с различными теплофизическими свойствами. Рассмотрим два случая, для чего изменим предыдущую задачу следующим образом.

Пусть пластина имеет зону из материала, имеющего значительно меньшую теплопроводность по сравнению с основной массой. Примем данные значения 0,015 Вт/(м.К) и 1,5 Вт/(м·К) соответственно. Остальные параметры задачи оставим прежними.

В первом случае аномальная зона имеет прямоугольную форму (рис. 4а), а во втором она образует своего рода теплоизолирующую полосу, разделяющую пластину на две части (рис. 4б).

Из рисунка 4 видно, что температура аномального участка резко выделяется на общем тепловом поле, которое в данном случае неравномерное. Кроме того, сам процесс прогрева происходит медленнее, чем в предыдущем примере.

Обсуждая полученные результаты, можно указать, что принятая в квазилинейной модели пропорциональная зависимость удельной мощности внутренних источников теплоты от температуры (11) является слишком грубым приближением при формализации законов теплопереноса при горении. Кроме того, в предыдущих примерах коэффициент теплопроводности принимался постоянной величиной, хотя в реальности он существенно зависит от температуры. Эти обстоятельства позволяют перейти к существенно нелинейной постановке задачи.

\section{Пример 4.}

Во-первых, следует учесть изменение коэффициента теплопроводности при увеличении температуры. Для этого будем рассчитывать его следующим образом:

$$
\lambda(T)=\lambda_{0} \alpha T,
$$

где $\lambda_{0}-$ начальная теплопроводность материала; $\alpha$ - константа.

Во-вторых, в реальных условиях при ламинарном диффузионном горении часто присутствуют эндотермические эффекты, связанные с влиянием на процесс образующихся продуктов горения [18]. Поэтому вместо выражения (11) введем нелинейный закон изменения мощности объемного источника $[19,20]$ :

$$
\gamma(T)=k T-\beta T^{3},
$$

где $\beta$ - константа. 


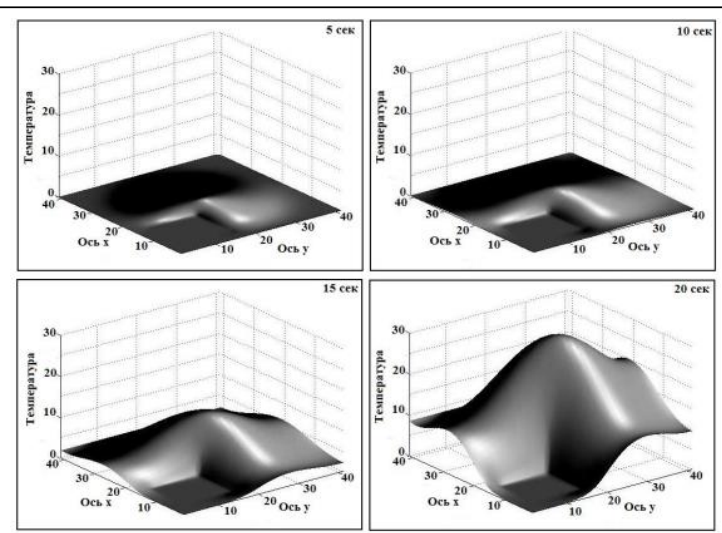

a)
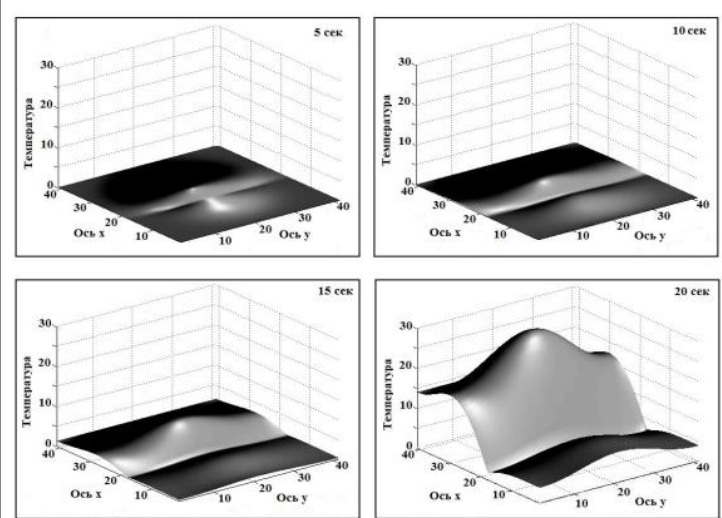

б)

Рис. 4. Результаты исследования квазилинейной модели передачи тепла в пластине:

a) с зоной аномальной теплопроводности,

б) с теплоизолирующим участком

Fig. 4. The study results of a quasilinear model of heat transfer in a plate:

a) with anomalous thermal conductivity zone,

б) with an insulating section

Также предусмотрим отдачу тепла в окружающую среду, температуру которой примем постоянной.

Результаты моделирования при $k=0,025$, $\alpha=0,05, \beta=0,01$ показаны на рисунке 5 .

Данные, представленные на нем, значительно отличаются от полученных ранее. Прежде всего видно, что процесс распространения теплоты проходит достаточно ярко выраженным широким фронтом. Кроме того, по мере развития процесса рост температуры замедляется, и она стремится к предельному значению. В реальных условиях такая ситуация может иметь место, например, при выгорании топлива.
В целом можно отметить, что приведенные результаты, полученные с использованием системы клеточных автоматов, вполне соответствуют принятым представлениям о протекании рассмотренных процессов.

Завершая рассмотрение примеров, важно отметить, что моделирующая программа во всех случаях подвергалась самым минимальным изменениям. Менялись только зависимости, по которым рассчитывались характеристики материала и при необходимости вводились координаты аномальных зон. При этом имитационный алгоритм оставался без изменений. Этот момент является очень ценным преимуществом использования дискретных методов компьютерного моделирования.

\section{Обсуждение и выводы}

Описанный в данной работе дискретный подход, использующий модели в виде систем клеточных автоматов, представляется более простым в реализации с точки зрения практического использования и физически более ясным по сравнению с применением дифференциальных уравнений с частными производными.

Следует отметить, что опубликованы результаты исследований с применением дискретного моделирования для анализа и других процессов химической технологии, таких как диффузия, конвективный теплоперенос, течение жидкостей и газов, деформирование твердых тел и пр. Перечисление ссылок на источники выходит за рамки данной статьи, но сле-

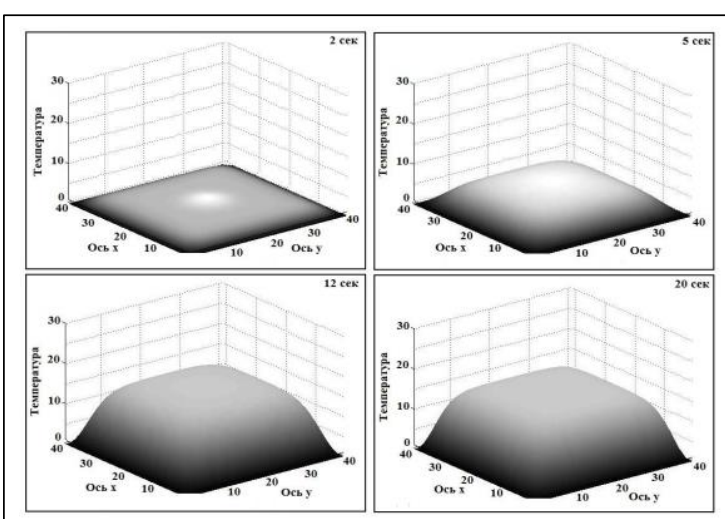

Рис. 5. Результаты исследования нелинейной модели передачи тепла, учитывающей эндотермические эффекты

Fig. 5. The study results of a nonlinear heat transfer model that takes into account endothermic effects 
дует отметить, что во всех случаях авторами получены весьма обнадеживающие результаты.

Принципиальное отличие между классическим и использованным подходами состоит в том, что пошаговый характер функций переходов клеточно-автоматной модели позволяет выразить присущую большинству физико-химических процессов нелинейность и дискретность самым непосредственным образом - локальными взаимодействиями.

Рассмотренные примеры призваны лишь отразить возможности дискретных моделей. Безусловно, для имитации процессов в реальных условиях следует переходить к объемным, трехмерным моделям. Однако такой переход вызовет лишь увеличение количества вычислений, при этом логика построения модели останется неизменной.

Резюмируя сказанное, можно отметить следующие основные достоинства применения клеточных автоматов при моделировании химико-технологических процессов.

1. Клеточными автоматами может быть описана любая форма границ исследуемой области, при этом постановка задачи практически не усложняется.

2. Так как функции перехода вида (1), определяющие поведение отдельных клеток, являются локальными, не возникает проблем при описании изменения свойств веществ во времени и пространстве. Это же касается и моделирования процессов в неоднородных средах.

3. Применение клеточных автоматов существенно облегчает анализ разрывных решений на границах областей.
Таким образом, клеточные автоматы позволяют описывать сложные механизмы процесса, которые другими методами описать затруднительно. С этих позиций дискретные подходы вообще и клеточные автоматы в частности можно рассматривать как представление задачи, которая ставит своей целью разбиение большой задачи на множество дискретных, мелких задач таким образом, что формулировка задачи для одного элемента одновременно является формулировкой всей задачи для всех элементов.

Вполне естественно, что клеточные автоматы имеют и недостатки.

Главным из них следует считать необходимость использования значительных вычислительных ресурсов, поскольку для получения основательных результатов требуется большое количество элементов, составляющих клеточный автомат, и, следовательно, заметный объем вычислений.

Однако данный недостаток может быть компенсирован следующим обстоятельством. Модели в виде клеточных автоматов по своей идеологии и архитектуре идеально подходят для успешного использования специализированных компьютеров, использующих технологии параллельных вычислений. Поэтому, учитывая стремительный рост возможностей средств компьютерной поддержки, клеточные автоматы в определенном смысле можно рассматривать как базовые математические модели. Несмотря на то, что сами они весьма просты, из них как из элементарных составляющих можно строить модели более сложных процессов и систем.

\section{Лumepamypa}

1. Баранов Д.А., Вязьмин А.В., Гухман А.А., Дильман В.В. Основы теории процессов химической технологии. В кн.: Процессы и аппараты химической технологии. М.: Логос, 2000. Т. 1. 480 с.

2. Wolfram S. A New Kind of Science. Wolfram Media Publ., 2002, 1197 p.

3. Таганов И.Н. Моделирование процессов массо- и энергопереноса. Нелинейные системы. Л.: Химия, 1979. 208 с.

4. Лобанов А.И., Петров И.Б. Математическое моделирование нелинейных процессов. М.: Юрайт, 2019. $255 \mathrm{c}$.

5. Бандман О.Л. Мелкозернистый параллелизм в математической физике // Программирование. 2001. № 4. C. 12-25.

6. Бандман О.Л. Отображение физических процессов на их клеточно-автоматные модели // Вестн. Томского гос. ун-та. Управление, вычислительная техника и информатика. 2008. № 2. С. 5-17.

7. Малинецкий Г.Г., Митин Н.А., Науменко С.А. Нанобиология и синергетика. Проблемы и идеи. Ч. 2: препр. ИПМ им. М.В. Келдыша. 2005. № 81.

8. Гусев А.В., Малинецкий Г.Г., Торопыгина С.А. Прикладная математика - проблемы и перспективы: препр. ИПМ им. М.В. Келдыша. 2013. № 44. 23 с.

9. Wolfram S. Statistical mechanics of cellular automata. Reviews of Modern Physics, 1983. vol. 55, no. 3, pp. 601-644. DOI: 10.1103/RevModPhys.55.601.

10. Аладьев В.3. Классические однородные структуры. Клеточные автоматы. Fultus Books, 2009. 535 с. 
11. Бобков С.П. Моделирование основных процессов переноса с использованием клеточных автоматов // Изв. вузов: Хим. Хим. технология. 2009. Т. 52. № 3. С. 109-114.

12. Тоффоли Т., Марголус Н. Машины клеточных автоматов; [пер. с англ.]. М.: Мир, 1991. 280 с.

13. Малинецкий Г.Г., Потапов А.Б., Подлазов А.В. Нелинейная динамика: Подходы, результаты, надежды. 2016. 280 с.

14. Бусленко Н.П. Моделирование сложных систем. М.: Наука, 1978. 399 с.

15. Колесов Ю.Б., Сениченков Ю.Б. Моделирование систем. Динамические и гибридные системы. СПб: БХВ-Петербург, 2012. 224 с.

16. Бандман О.Л. Клеточно-автоматные модели пространственной динамики // Системная информатика. 2006. Т. 10. С. 59-113.

17. Бобков С.П. Использование дискретных подходов для моделирования основных процессов химической технологии // Российский химический журнал. 2019. Т. 63. № 3-4. С. 22-30.

18. Полежаев Ю.В. Законы горения. М.: Энергомаш, 2006. 352 с.

19. Курдюмов С.П., Малинецкий Г.Г., Потапов А.Б., Самарский А.А. Структуры в нелинейных средах. В кн.: Компьютеры и нелинейные явления. М.: Наука, 1988. С. 5-43.

20. Бандман О.Л. Режимы функционирования асинхронных клеточных автоматов, моделирующих нелинейную пространственную динамику // Прикладная дискретная математика. Дискретные модели реальных процессов. 2015. № 1. С. 105-119.

\title{
Simulation of the heat conduction process using cellular automata systems
}

\author{
S.P. Bobkov ${ }^{1}$, Dr.Sc. (Engineering), Professor, bsp@isuct.ru \\ E.G. Galiaskarov ${ }^{1}$, Ph.D. (Chemistry), Associate Professor \\ ${ }^{1}$ Ivanovo State University of Chemical Technology, Ivanovo, 153000, Russian Federation
}

\begin{abstract}
The paper concentrates on the use of discrete dynamic models as an alternative to the classical methods of studying thermal processes in chemical technology.

An adequate description of heat transfer phenomena is a hugely important problem, both in theoretical terms and from the standpoint of the practical use of thermal processes. In addition, modern teaching methods require the introduction of electronic textbooks, virtual laboratory workshops, simulation programs, which also need a correct description of the phenomena under study.

The classical approach to modeling heat transfer in a continuous medium involves the use of heat conduction equations, in which the thermophysical characteristics of materials are usually constants. Taking into account the effect of temperature on the characteristics of materials leads to the need to study nonlinear equations, which causes significant computational difficulties. In this regard, it becomes expedient to use fundamentally different approaches to modeling thermal conductivity, one of which is models based on systems of cellular automata.
\end{abstract}

Discrete dynamic models in the form of deterministic cellular automata systems are used. In this case, a continuous medium is considered as a set of interacting elements whose behavior is completely described by local functions. The paper describes the main approaches and general methodology for the development of discrete models.

The examples of cellular automata systems use for simulation of nonlinear heat transfer processes are considered, taking into account the heterogeneity of the material and the presence in the material of volumetric sources of variable power in it.

The obtained data of discrete simulation are in good agreement with the results of using the classical approach and do not contradict the generally accepted views adopted in the theory of thermal phenomena. The paper shows the features of the discrete approach in comparison with the use of partial differential equations with nonlinear coefficients, shows the advantages and disadvantages.

Keywords: discrete simulation; cellular automata; heat transfer; nonlinear heat conduction problems; volumetric heat sources. 


\section{References}

1. Baranov D.A., Vyazmin A.V., Gukhman A.A., Dilman V.V. Foundations of the theory of processes of chemical technology. In Processes and Devices of Chemical Technology. Moscow, 2000, vol. 1, 480 p. (in Russ.).

2. Wolfram S. A New Kind of Science. Wolfram Media Publ., 2002, 1197 p.

3. Taganov I.N. Modeling Mass and Energy Transfer Processes. Non-Linear Systems. Leningrad, 1979, 208 p. (in Russ.).

4. Lobanov A.I., Petrov I.B. Mathematical Modeling of Nonlinear Processes. Moscow, 2019, 255 p. (in Russ.).

5. Bandman O.L. Fine-grained parallelism in mathematical physics. Programmirovanie, 2001, no. 4, pp. 12-25 (in Russ.).

6. Bandman O.L. Simulating physical processes by cellular automata. Tomsk State University Journal of Control and Computer Science, 2008, no. 2, pp. 5-17 (in Russ.).

7. Malinetskii G.G., Mitin N.A., Naumenko S.A. Nanobiology and Synergetics. Problems and Ideas. Pt. 2. KIAM Preprint, 2005, no. 081 (in Russ.).

8. Gusev A.V., Malinetskiy G.G., Toropygina S.A. Applied mathematics - problems and prospects. KIAM Preprint, 2013, no. 44, 23 p. (in Russ.).

9. Wolfram S. Statistical mechanics of cellular automata. Reviews of Modern Physics, 1983, vol. 55, no. 3, pp. 601-644. DOI: 10.1103/RevModPhys.55.601.

10. Aladjev V.Z. Classical Homogeneos Structures. Cellular Automata. Fultus Publishing, 2009, 535 p. (in Russ.).

11. Bobkov S.P. Simulation of transfer basic processes with using cellular automata. Izvestiya Vysshikh Uchebnykh Zavedenii: Khimiya i Khimicheskaya Tekhnologiya, 2009, vol. 52, no. 3, pp. 109-114 (in Russ.).

12. Toffoli T., Margolus N. Cellular Automata Machines. The MIT Press publ., 1987, 276 p. (Russ. ed.: Moscow, 1991, 280 p.).

13. Malineckii G.G., Potapov A.B., Podlazov A.V. Nonlinear Dynamics: Approaches, Results, Hopes. 2016, 280 p. (in Russ.).

14. Buslenko N.P. Modeling Complex Systems. Moscow, 1978, 356 p. (in Russ.).

15. Kolesov Yu.B., Senichenkov Yu.B. System Modeling. Dynamic and Hybrid Systems. St. Petersburg, 2012, 224 p. (in Russ.).

16. Bandman O.L. Cellular automata models of spatial dynamics. System Informatics, 2006, vol. 10, pp. 59-113 (in Russ.).

17. Bobkov S.P. Use of discrete approaches for simulation the basic processes of chemical technology. Russian Chemistry Journal, 2019, vol. 63, no. 3-4, pp. 22-30 (in Russ.).

18. Polezhaev Yu.V. Combustion Laws. Moscow, 2006, 352 p. (in Russ.).

19. Kurdyumov S.P., Malineckii G.G., Potapov A.B., Samarsky A.A. Structures in nonlinear media. In: Computers and Non-Linear Phenomena. Moscow, 1988, pp. 5-43 (in Russ.).

20. Bandman O.L. Functioning modes of asynchronous cellular automata simulating nonlinear spatial dynamics. Applied Discrete Mathematics, 2015, no. 1, pp. 105-119 (in Russ.).

\section{Для цитирования}

Бобков С.П., Галиаскаров Э.Г. Моделирование процесса теплопроводности с использованием систем кцеточных автоматов // Программные продукты и системы. 2020. Т. 33. № 4. C. 641-650. DOI: 10.15827/0236-235X.132.641-650.

\section{For citation}

Bobkov S.P., Galiaskarov E.G. Simulation of the heat conduction process using cellular automata systems. Software \& Systems, 2020, vol. 33, no. 4, pp. 641-650 (in Russ.). DOI: 10.15827/0236235X.132.641-650. 